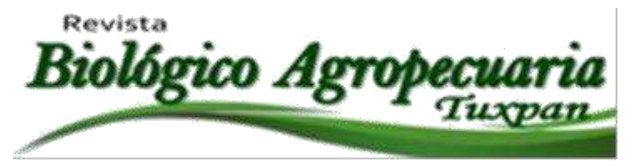

\title{
Estado del arte Phymatotrichopsis omnivora (SHEAR) duggar, revisión y actualidad
}

State of the art Phymatotrichopsis omnivora (SHEAR) duggar, review and present

\author{
Martínez-Escudero Lorenza E. ${ }^{1}$, Melgoza-Castillo Alicia ${ }^{2}$, Pérez-Leal Ramona ${ }^{3 凶}$, Ortega- \\ RodríguezAnabel $^{3}$ y Villalobos-Pérez Elizabeth ${ }^{3}$
}

1 Estudiante de posgrado de la Facultad de Zootecnia, Universidad Autónoma de Chihuahua. 2 Profesor de la Facultad de Zootecnia, Universidad Autónoma de Chihuahua.

Periférico Francisco R. Almada, Km 1 de la Carretera Chihuahua-Cuauhtémoc. Chihuahua, Chih., México, 31031. Tel. (614) 434-0303. 3 Profesor de la Facultad de Ciencias Agrotecnológicas, Universidad Autónoma de Chihuahua Campus I, Chihuahua, Chih., México. Tel. (614) 4391810

${ }^{\circledR}$ Autor para correspondencia: perezleal@hotmail.com

Recibido: 19/01/2016

Aceptado: 28/06/2016

\section{RESUMEN}

Phymatotrichopsis omnivora es un hongo que ocasiona la pudrición de la raíz siendo considerada una de las más serias enfermedades de las plantas debido al amplio rango de hospedantes que afecta, lo que conlleva a grandes pérdidas económicas. Son varios factores son que influyen en el desarrollo de la enfermedad y la sintomatología puede variar de planta a planta. Este hongo coloniza primeramente las células corticales y forma una red que cubre la superficie de las raíces, bloqueando el sistema vascular así como el movimiento del agua en la planta. Los métodos de control químico son costosos y poco eficientes, por lo que se buscan otros métodos como la utilización de plantas resistentes, la aplicación de organismos antagonistas, o el uso de ciertos extractos vegetales, los cuales han demostrado tener cierta actividad en el control de este patógeno.

Palabras clave: control biológico; esclerocios; enfermedad; sintomatología; pudrición de raíz.

\begin{abstract}
Phymatotrichopsis omnivora it's a fungi that causes root rot and it is considered one of the most serious diseases of plants because of the wide host range affecting, and causing great economic losses. Some factors have influence on disease development, and symptoms can
\end{abstract}


vary from plant to plant. This fungus colonizes primarily cortical cells and forms a blanket which covers the surface of the roots, blocking the vascular system and the movement of water too. Chemical control methods are costly and inefficient, so that other control methods are sought. Use of resistant plants as well as the application of antagonists as Trichoderma $s p$, or use of plant extracts has shown good activity against this pathogen, which they have shown some activity in the control of this pathogen.

Keywords: disease, Phymatotrichopsis omnivora, root rot, sclerotia, symptomatology.

\section{INTRODUCCIÓN}

En 1889 Pammel reportó por primera vez una enfermedad en el cultivo del algodón, la cual ocasionaba la pudrición de la raíz de la planta, por lo que esta enfermedad fue denominada pudrición de la raíz del algodonero. Esta enfermedad también recibe el nombre de "pudrición Texana" o "pudrición de la raíz por Phymatotrichum", debido al organismo causal.

\section{Phymatotrichopsis omnivora} (Duggar) Hennebert [sin. Phymatotrichum omnivorum (Shear) Duggar], es un hongo filamentoso habitante natural de los suelos alcalinos y con bajo contenido de materia orgánica. Es endémico del suroeste de los Estados Unidos y norte de México así como de algunas partes de Asia central (Percy, 1983; Goldberg, 1997; EPPO).

La pudrición de la raíz ocasionada por este hongo es considerada una de las más serias enfermedades de las plantas, debido al amplio rango de hospedantes y la destrucción de los cultivos que infecta, incluyendo cultivos de importancia económica como el algodón (Gossypium spp.), alfalfa (Medicago sativa), nogal entre otros), afectando a más de 2000 especies de plantas dicotiledóneas
(Agrios, 1997, Riggs, 2008; EPPO Data Sheets on Quarantine Pests).

Desde su descubrimiento se han desarrollado numerosas investigaciones encaminadas a de detener o controlar el ataque de este hongo las cuales incluyen tanto en el control químico como en el control biológico.

El control químico se realiza mediante agentes antifúgicos selectivos para este hongo, los cuales inhiben tanto el crecimiento del micelio como el desarrollo de esclerocios, sin embargo éstos son difíciles de aplicar y no son económicamente rentables, por lo que es necesario un manejo integrado para el control de la enfermedad (VargasArispuro et al, 2006; Samaniego 2007).

Por otro lado, con el aumento de poblaciones microbianas en el suelo, se ha observado un beneficio en tanto en la reducción de la enfermedad como en la inhibición de la germinación de los esclerocios (Samaniego, 2008).

\section{DESARROLLO DEL TEMA TAXONOMÍA Y CICLO DE VIDA}

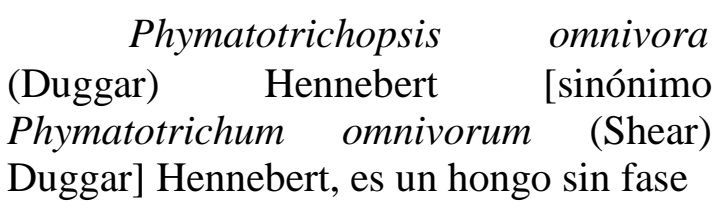


sexual conocida (Deuteromiceto), fue nombrado como Phymatotrichum omnivorum (Shear) basándose en las características conidiales, las cuales son producidas en tapetes de esporas que crecen cerca de la planta enferma (Duggar, 1916). Se encontraba dentro de la clase Hypomicetes, orden Hyphales (Moniliales), y fue asociado dentro del

Phylum Basidiomycota. Hennebert (1973) lo renombró como Phymatotrichopsis omnivora (Duggar) Hennebert, debido a la afinidad que observó que tenía este hongo con Botritys, dentro de la división Ascomicota.

Recientemente se ha utilizado la técnica de amplificación de secuencias de DNA ribosomal para construir el árbol filogenético en donde los resultados indican que $P$. omnivora debería ser colocado en el Orden Pezizales (discomicetos operculados) dentro de la división Ascomycota, y aunque las secuencias de rDNA, indican que existe similitud con Rhizinia ondulata, aún no hay datos concluyentes en su posición taxonómica (Marek 2005).

\section{BIOLOGÍA Y RANGO DE HOSPEDANTES}

La sobrevivencia y reproducción de este hongo se realiza mediante hifas o esclerocios en el suelo, siendo las hifas vegetativas el medio por el cual el hongo se mueve a través del suelo o sobre el tejido radicular. Se esparce lentamente de planta a planta cuando una hebra fúngica de una raíz infectada crece a través del suelo y alcanza una raíz sana (Agrios, 1998).
Presenta tres estados biológicos: micelio, conidias y esclerocios. Las hifas que constituyen el micelio son largas, septadas y poseen algunos núcleos, estas al entrelazarse forman cordones miceliales (rizomorfos) que presentan ramas laterales en forma de cruz (crucetas), principal característica de identificación del patógeno (Agrios, 1997), estos cordones miceliales pueden ser observados sobre las raíces infestadas. Los cordones miceliales o rizomorfos, es una de las formas de diseminación más importantes. Bajo condiciones de humedad, el hongo esporula sobre la superficie del suelo infectado observándose un tapete de micelio amarillento, en donde la masa de esporas está compuesta por hifas con conidióforos globosos, cortos, gruesos, simples o ramificados en cuyos extremos hinchados se producen grandes cantidades de esporas, sin embargo estas esporas no son capaces de germinar $u$ ocasionar enfermedad (Riggs 2008).

Por otro lado los esclerocios son estructuras de resistencia, es decir, son el medio primario que el organismo utiliza para sobrevivir al invierno, en ausencia de un hospedante susceptible o cuando las condiciones ambientales no son favorables (Agrios, 1998). Riggs (2008) menciona que los esclerocios logran sobrevivir por al menos cinco años en el suelo, mientras que Samaniego (2008), señala que la sobrevivencia es capaz de alcanzar hasta 20 años si no existen factores que los limiten, ya que pueden ser afectados por la flora circundante del suelo.

Hospedantes. En varias regiones de México este patógeno ocasiona la pudrición de las raíces plantas tanto silvestres como cultivadas y de árboles 
frutales de interés económico, como son los durazneros, pistachero, nogales, mango, entre otros, ocasionándoles la muerte o la disminución en la producción (Samaniego et al, 2001; Samaniego y Herrera, 2003; Ramírez y Sáinz, 2006).

\section{Generalmente}

las monocotiledóneas no son afectadas por $P$. omnivora, aunque es capaz de sobrevivir y propagarse en el tejido de este tipo de plantas. A pesar de que el micelio del hongo se encuentra en asociación con sus raíces, éstas no muestran signos de daño, únicamente una pequeña lesión en el sitio de contacto con el micelio del hongo (Rush et al, 1984a; Singleton et al, 2001; Riggs, 2008).

Desarrollo de la enfermedad. La severidad y presentación de los síntomas depende de factores como condiciones geográficas, ambientales, la época del año, la patogenicidad del hongo, y la edad y la susceptibilidad de la planta. (Ramírez y Sáinz, 2006; Tood-Watson et al., 2007, Samaniego et al, 2014), pero además las raíces producen exudados ricos en carbohidratos los cuales proporcionan la energía requerido para el desarrollo, $P$. omnivora (Rush et al. 1984). Se ha observado que las semillas de algodón empiezan a ser atacadas por el hongo 8 días después de la emergencia, presentando la raíz síntomas de decoloración y una apariencia aguanosa, la cual se presenta a las 24 horas después del inicio del contacto, y a partir de ahí el hongo se extiende sobre la raíz. La colonización de la raíz puede ocurrir 14 días antes de que los síntomas puedan ser observados (Rush et al, 1984b, 1984c). Por otro lado, se ha observado que en árboles de nogal cuando la densidad de plantación es alta, se presenta más mortandad de los árboles, no obstante, la presencia o desarrollo de la enfermedad se encuentra estrechamente asociada a la susceptibilidad de la planta al patógeno (Samaniego-Gaxiola et al, 2014).

El inóculo primario pueden ser los esclerocios o las hifas que se encuentran sobre las raíces de las plantas infectadas, los árboles enfermos pueden transmitir la enfermedad a los árboles contiguos sanos, ya que su diseminación puede ocurrir a través del contacto de las raíces infectadas con las raíces sanas (Samaniego et al, 2001). En estudios realizados en raíces de algodón afectadas por $P$. omnivora, se observó que el mayor número de cordones miceliales viables recuperados del suelo se presentaron durante los meses de agosto a septiembre a una profundidad de 15 a $60 \mathrm{~cm}$, es decir, cuando la incidencia de la enfermedad se presenta en mayores proporciones (Rush et al, 1984c)

La forma de penetración a la raíz aun no es clara, sin embargo el hongo coloniza el interior de las raíces obstruyendo el sistema vascular y bloqueando el movimiento del agua (Riggs, 2008).

Síntomas. Los síntomas de las plantas varían un poco de acuerdo al cultivo infectadas $\mathrm{y}$ no son visibles normalmente, como en el caso del algodón, que se observan solo hasta que alcanza la etapa de floración, los síntomas foliares no aparecen en plantas jóvenes hasta los 25 días después de la emergencia, en comparación con las plantas de más edad, en las cuales los síntomas aparecen a los 12 días después de la inoculación (Rush et al, 1984c), mientras que en el caso del manzano se requiere de un período de 9 a 12 semanas para que se presenten síntomas, pero en 
términos generales, el primer efecto visible es la marchitez del follaje, el cual presenta una coloración amarillenta al principio y cambia al final a café bronceado, (Castro y Rodríguez, 1970; Ramírez y Sáinz, 2006). Los síntomas en general incluyen el colapso del tejido infectado de la raíz (pudrición), con un rápido marchitamiento de las hojas las cuales mueren quedando adheridas al árbol en la mayoría de los casos, posteriormente ocurre el deceso de la planta (Riggs, 2008). Los cordones miceliales característicos del hongo, se pueden observar sobre la superficie de las raíces infectadas.

La infección y muerte de los cultivos estacionales ocurre principalmente durante el inicio de la estación, lo cual quiere decir que el hongo infecta a bajas temperaturas, solo que requiere de más tiempo para hacerlo. El hongo sobrevive al invierno como cordones miceliales sobre la superficie de raíces perennes y cuando las temperaturas son suficientes el hongo inicia su crecimiento (Lyda y Burnett, 1971).

Upalapati et al, 2009, utilizando imágenes confocales de las interacciones de $P$. omnivora con las raíces de Mediccago truncatula, encontraron que el micelio del hongo no forma ninguna estructura especializada en la penetración, sino que coloniza primordialmente las células corticales y forma un manto que cubre la superficie de las raíces. Al entrar en contacto con éstas, la hifa del hongo crece formando un manto a lo largo de las estrías de la raíz, entre las células epidérmicas, y posteriormente se desarrolla en los espacios intracelulares, infectando las células epidermales y corticales a los cinco días después de la inoculación.

\section{FACTORES QUE INFLUYEN EN EL PROCESO DE INFECCIÓN}

Como se mencionó anteriormente, la infección puede depender de la época del año, de la patogenicidad del hongo y de la susceptibilidad de las plantas a éste, de la temperatura, (Ramírez y Sáinz, 2006; Todd-Watson et al., 2007), la densidad del inóculo o número de esclerocios en el suelo también son factores importantes para el desarrollo de la enfermedad, encontrándose entre 125 a 625 esclerocios por gramo de suelo (Lyda y Burnett, 1970). Otros factores que se encuentran relacionados con el incremento de la enfermedad son el número de riegos, abundante precipitación; profundidad de las raíces y el manejo del cultivo (SamaniegoGaxiola et al, 2001; Samaniego-Gaxiola et al, 2014).

El medioambiente del suelo es determinante para el desarrollo del proceso de infección de los patógenos de las plantas. La composición del suelo se encuentra relacionada con el metabolismo de las raíces y con las transformaciones metabólicas de los elementos minerales del suelo realizadas por los microorganismos asociados a las raíces de las plantas (Junhke et al., 1987). Las actividades microbianas juegan un papel muy importante en los cambios que ocurren en el suelo como son la concentración de oxígeno, de bióxido de carbono y carbonatos, Las repercusiones en las variaciones en la concentración de estos gases, se manifiestan en el desarrollo o en la inhibición de los microorganismos incluyendo a los patógenos como Phymatotrichopsis omnivora. Este hongo se ve favorecido por niveles elevados en suelo de $\mathrm{CO}_{2} \mathrm{o}$ 
$\mathrm{HCO}_{3}$, debido a que se aumenta el $\mathrm{pH}$ de éste, lo que puede beneficiar altamente a la germinación de esclerocios en caso de que éstos se encuentran presentes (Singleton, et al, 2001; Samaniego, 2008b, Samaniego, 2008c).

Niveles de $\mathrm{pH}$ de entre 6 y 8 , es decir, el pH que se encuentra en suelos calcáreos alcalinos, favorecen la germinación de esclerocios y desarrollo del hongo. (Gunasekaran, 1927; Samaniego, 2008a;).

De igual forma, la composición del suelo influye sobre la capacidad metabólica del sistema radicular de las plantas, la toma de nutrientes y la absorción de agua. Un balance inadecuado o fluctuaciones en la composición de los factores del suelo pueden afectar el metabolismo de la planta, lo que la hace susceptible a enfermedades radiculares debidas al ataque por patógenos. (Singleton, et al, 2001; Samaniego, 2008a).

Las repercusiones en las variaciones en la concentración de los gases mencionados anteriormente, se manifiestan en el desarrollo o en la inhibición de los microorganismos incluyendo a los patógenos como Phymatotrichopsis omnivora. Este hongo se ve favorecido por niveles elevados en suelo de $\mathrm{CO}_{2}$ o $\mathrm{HCO}_{3}$, debido a que se aumenta el $\mathrm{pH}$ de éste, lo que puede beneficiar altamente a la germinación de esclerocios en caso de que éstos se encuentran presentes (Gunasekaran, 1927; Singleton, et al, 2001; Samaniego, 2008c).

Se ha encontrado una correlación entre el desarrollo del patógeno y el tipo de suelo, ya que su desarrollo se ve favorecido en suelos de tipo vertisol o en suelos limosos (Percy, 1983; Ramírez y Sáinz, 2006). Por otro lado el contenido de sodio parece tener influencia en la producción de esclerocios, ya que no es común encontrar pudrición de la raíz en suelos con niveles de sodio intercambiable mayores que 500 ppm. (Riggs, 2008), en cuanto a la concentración en el suelo de otros cationes como el potasio, calcio y magnesio, no parece tener influencia sobre el desarrollo de $P$. omnivora (Mueller et al., 1983).

La temperatura del suelo es importante tanto para la sobrevivencia y el tiempo de desarrollo de la pudrición de la raíz, como para la producción de esclerocios. Se ha observado formación de esclerocios a temperaturas de entre 15 y $30^{\circ} \mathrm{C}$, siendo $28^{\circ} \mathrm{C}$ la temperatura óptima tanto de crecimiento del micelio como para el desarrollo de la infección, es decir para la pudrición de la raíz (Gunasekaran, 1927; Lyda y Burnett, 1971; Percy, 1983).

Importancia económica. Este hongo es probablemente el que tiene una mayor gama de hospedantes, afectando a más de 2000 especies de dicotiledóneas, incluyendo cultivos de importancia económica como algodón (Gossypium hirsutum L.), alfalfa (Medicago sativa $\mathrm{L}$ ) vid (Vitis pp), nogal (Carya illinoensis), pistacho (Pistacia sp) y durazno (Prunus pérsica), entre otros. (Goldberg, 1997; Samaniego et al, 2001, Samaniego y Herrera, 2003; Samaniego et al, 2003).

Métodos de control. $P$. omnivora es un patógeno difícil de eliminar, ya que como se mencionó anteriormente, los esclerocios son capaces de permanecer en estado latente en el suelo durante muchos años (Rush et al., 1984). A la 
fecha no se ha encontrado un método de control que resulte efectivo y económicamente rentable, aun la búsqueda de plantas resistentes a la infección por este patógeno ha resultado difícil (Percy and Rush, 1984, Cook et al., 1995, Tarango, 2006).

Los principales método de control están basado en la rotación de cultivos, en el manejo del suelo conocido como "tratamiento Arizona modificado Laguna Seaca" (Castro, 2001), eliminación de la planta enferma, la adición de abonos que además de disminuir el $\mathrm{pH}$ del suelo, al descomponerse favorecen la proliferación de poblaciones de microorganismos que pueden presentar antagonismo al hongo (Agrios, 1998).

Control químico. Los fungicidas utilizados además de ser costosos deben ser aplicados cada año, ya que el micelio y los esclerocios del hongo tienen diferente susceptibilidad, por otro lado la influencia de factores como el $\mathrm{pH}$

(Samaniego-Gaxiola, 2008c), y las características del suelo influyen en la efectividad de los fungicidas en la infección de la raíz. (Rush y Lyda, 1982). Estudios in vitro han demostrado la muerte del micelio o los esclerocios con la aplicación al medio de cultivo de ácidos grasos volátiles (SamaniegoGaxiola, 2013).

Control biológico. Los estudios relacionados con el control biológico en contra de $P$. omnivora están basados en el uso de extractos vegetales (VargasArispuro et al., 2005); aceites esenciales producidos por plantas como el orégano (Valero et al., 2005), o aceites extraídos de semillas oleaginosas los cuales presentan cierta actividad antifúngica con una significante reducción en el crecimiento de las hifas de este patógeno o de una completa inhibición en el desarrollo de los esclerocios, (Hu et al., 2011).

En estudios relacionados con microorganismos capaces de inhibir el crecimiento de $P$. omnivora, se ha encontrado que bacterias y actinomicetos capaces de inhibir el desarrollo de esclerocios (Zuberer et al., 1988). Por otro lado, el hongo Trichoderma sp es un agente ampliamente utilizado como biocontrol debido a su capacidad de producir enzimas hidrolíticas que atacan a hongos fitopatógenos, y es capaz de invadir los esclerocios de $P$. omnivora impidiéndoles su germinación, ya que durante la interacción entre ambos hongos se estimula la expresión de mecanismos enzimáticos de Trichoderma (Samaniego, 2008: Cervantes-Martínez et al., 2010; Guigón-López et al., 2014; GuigónLópez et al., 2015).

Los microorganismo del suelo juegan un papel importante en el control de patógenos (Samaniego-Gaxiola, 2007), las raíces de las plantas, mediante la producción de exudados, proporcionan un hábitat ideal para el desarrollo de microorganismos con los que las raíces establecen asociaciones, ya sea para el intercambio de nutrientes, estimulo del crecimiento vegetal o protección contra patógenos, entre otros (Whipps, 2001; Dakora y Phillips, 2002; Sarabia et al, 2010).

\section{CONCLUSIONES}

La utilización de fungicidas además de contaminar el suelo, no solamente atacan al patógeno, sino que eliminan a organismos nativos que pueden ejercer algún biocontrol en contra 
de los patógenos (Whipps, 2001), por lo que es de suma importancia contar con agentes biológicos que puedan controlar a los patógenos.

Existen infinidad de microorganismos en el suelo que conviven con $P$. omnivora y que pueden tener algún efecto antagónico sobre éste patógeno y las raíces de las plantas proporcionan un hábitat ideal para el

desarrollo de todo tipo de microorganismos, no solamente de patógenos, existiendo un gran número de microorganismos con los que se establecen asociaciones para diversas actividades como son el intercambio de nutrientes, el estímulo del crecimiento vegetal o la protección contra patógenos, entre otros, y con beneficios para ambos.

El estudio del suelo y de la rizósfera son grandes áreas de

oportunidad para continuar con investigaciones en la búsqueda de antagonistas para el control de $P$. omnivora.

\section{LITERATURA CITADA}

Agrios, G.N. 1997. PLANT PATHOLOGY. 4th Edition. Academic Press (AP) Publisher. Florida, USA. 803 p.

Alderman, S, C. and R. B. Hine 1982. Vertical distribution in soil of and induction of disease by strands of Phymatotrichum omnivorum. The American Phytopathological Society 72:409-412. Castro, F. J. y V. A. Rodríguez. 1970. Pruebas preliminares para el combate de la pudrición texana del durazno en el bajío. Instituto Nacional de investigaciones agrícolas SAG. Circular CIAB No. 34. https://doi.org/10.1094/Phyto-72-4

Casto, M. R. 2001. Como eliminar los síntomas de pudrición texana en el nogal. 9o. Simposio Internacional Nogalero. Raíces del Nogalero Organizado: Manejo de Agua, Inocuidad y Comercialización". Torreón, Coah.

Cervantes-Martínez R., V. HernándezHernández, G. GonzálezCervantes, E. Favela-Chávez, y V.de P. Álvarez-Reyna. 2010. Antagonismo de cepas nativas de Trichoderma sp. aisladas en la Comarca Lagunera contra Phymatotrichum omnivorum (Shear) Duggar. Revista Agraria Nueva Epoca- Año VII · Vol. 7 . No. 1, 2, 3 .

Chitrampalam, P. and M. W. Olsen. 2014. Research Report. Genetic diversity and fungicide sensitivity of Phymatotrichopsis omnivora isolates from cotton in Arizona. College of Agriculture and Life Sciences. 12p.

Cook, C. G., J. L. Riggs, J. R. Samrt and B. A. Mullin-Scharding. 1995. Evaluation of six Kenaf cultivars for resistance to Phymatottichum omnivorum. Industrial Crops and Products. 4. 229-232. https://doi.org/10.1016/0926-6690(

Dakora, D.F. y D. A. Phillips. 2002. Root exudates as mediators of mineral acquisition in low-nutrient environments. Plant and Soil. 245: 35-47. https://doi.org/10.1023/A:1020809 
Duggar, B. M. 1916. The Texas root rot fungus and its conidial stage. Annals of the Missouri Botanical Garden 3: 11-23. https://doi.org/10.2307/2990071

EPPO quarantine pest. Prepared by CABI and EPPO for the EU under Contract 90/399003. Data Sheets on quarantine pests. Phymatotrichopsis omnivora. http://www.eppo.org/QUARANTI NE/fungi/Phymatotrichopsis_omn ivora/PHMPOM_ds.pdf.

Guigón.López, C., V. Guerrero-Prieto, S. Lanzuise y M. Lorito. 2014. Enzyme activity of extracellular protein induced in Trichoderma asperellum and $T$. longibrachiatum by substrates based on Agaricus bisporus and Phymatotrichopsis omnivora. Fungal Biology. 118: 211-221. https://doi.org/10.1016/j.funbio.20 Guigón.López, C., F. Vargas-Albores, V. Guerrero-Prieto, M. Ruocco y M. Lorito. 2015. Changes in Trichoderma asperellum enzyme expression during parasitism of the cotton root rot pathogen Phymatotrichopsis omnivora. Fungal Biology. 119: 264-273. https://doi.org/10.1016/j.funbio

Goldberg, N. P. 1997. Root Diseases of Pistachio Trees in New Mexico Extension Plant Pathologist. New Mexico State University. Guide H647.

Gunasekaran, M. 1927. Physiological studies on Phymatotrichum omnivorum iv. Effect of ph and the interaction of temperature, minerals and carbon source on growth in vitro. Mycopattlologia et Mycologia applicata, vol. 50, (4): 313-321.

https://doi.org/10.1007/BF0205003
Hennebert, G.L. 1973. Botrytis and Botrytis-like genera. Persoonia. 7, 183-204.

Hu, P., A. S. Wang, A. S. Engledow, E. B. Hollister, K. L. Rothlisberger, J. E. Matocha, D. A. Zuberer, T. L. Provin, F.M. Hons and T.J. Gentry. 2011. Inhibition of the germination and growth of Phymatotrichopsis omnivora (cotton root rot) by oilseed meals and isothiocyanates. Applied Soil Ecology. 49: 68-75.

https://doi.org/10.1016/j.apsoil.201

Junhke, M.E., D. E. Mathre, S. C. Sands.

1987. Identification and Characterization of RhizosphereCompetent Bacteria of Wheat. Applied Environmental Microbiology. 53: (12): 2793 2799. https://doi.org/10.1128/AEM Lyda S.D and E. Burnett, 1971. Influence of temperature on Phymatotrichum sclerotial formation and disease development. Phytopathology 61:728-730. https://doi.org/10.109

Lyda, S. D. and Burnett E. 1970. Sclerotial inoculums density of Phymatotrichopsis omnivoum and development of Phymatotrichym root rot in cotton. Phytopatology. 60:729-731.

https://doi.org/10.1094/Phyto-60-7 https://doi.org/10.1094/Phyto-60-7 Marek, S. 2005. Molecular phylogeny of Phymatotrichopsis omnivora. Phytopathology 95:S65. APS Annual Meeting Abstracts of Presentations, Austin, Tx. Vol. 95, No. 6 (Supplement), S1. https://doi.org/10.1094/PHYTO.20 Mueller, J. P., R. B. Hine, D. A. Pennington and S. J. Ingle. 1983. Relationship of soil to the 
distribution of Phymatotrichum omnivorum. The American Phytopathological Society. Vol. 73:1364-3168.

https://doi.org/10.1094/Phyto-73-1

Pammel, L.H. 1889. Cotton root-rot.

Texas Agricultural Experiment

Station Bulletin 7: 5-30.

https://repository.tamu.edu/handl e/1969.1/2851).

Percy, R. G. 1983. Potential range of Phymatotrichum omnivorum as determinded by edaphic factors. Plant Disease. 981-983. https://doi.org/10.1094/PD-67-981

Percy, R. G. and C. M. Rush. 1985. Evaluation of four upland cotton genotypes for a rate-limiting resistance to Phymatotrichum root rot. The American Phytopathological Society. 75(4):463-466. https://doi.org/10.1094/Phyto-75-4

Ramírez, V. J. y R. A. Sáinz. 2006. Hospedantes y distribución de la "Pudrición Texana" (Phymatotrichum omnivorum) en Sinaloa. Tercer Foro Estatal de Ciencia y Tecnología. Sinaloa, Investigación para el desarrollo. Culiacán, Sinaloa, 8 y 9 de diciembre. www.agrobiologica.com.mx.

Riggs, L. J. 2008. Historical information on Phymatotrichopsis omnivora (Duggar) Hennebert the root rot fungus. Beltwide Cotton Conferences, Nashville, Tennessee.Pp.199-200. http://ncc.confex.com/ncc/2008/te chprogram/S1672.HTM

Rush, C. M. and D. Lyda. 1982. Effects of anhydrous ammonia on mycelium and sclerotia of Phymatotrichum omnivorum.

Phytopathology 72:1085-1079. https://doi.org/10.1094/Phyto-7
Rush, C. M., T. J. Gerik and S. D. Lyda, 1984a. Interactions between Phymatotrichum omnivorum and Sorghum bicolor. Plant Disease 68:500-501.

https://doi.org/10.1094/PD-69-500

Rush, C. M., Lyda, S. D., and T. J. Genik. 1984b. The relationship between time of cortical senecence and foliar symptom development of Phymatotrichum root rot of cotton. Phytopathology. 74 (12):14641466. https://doi.org/10.1094/Phyto-74-1

Rush, C..M., D. R. Upchurch, and T. J. Genik. 1984c. In situ observations of Phymatotrichum omnivorum with a borescope mini-rhizotron system. Phytopathology. 74:104105. https://doi.org/10.1094/Phyto-74-1

Samaniego-Gaxiola, J. A. 2007. Perspectivas de la investigación de Phymatotrichopsis omnivora y la enfermedad que causa. Agricultura Técnica de México. 33 (3):309-318.

Samaniego-Gaxiola, J. A. 2008a. Germinación y sobrevivencia de esclerocios de (Phymatotrichopsis omnivora en respuesta a $\mathrm{NaOCl}$ y suelo con glucosa. Agricultura Técnica de México. 34 (4): 375385.

Samaniego-Gaxiola, J. A. 2008b. Efecto del $\mathrm{pH}$ en la sobrevivencia de esclerocios de Phymatotrichopsis omnivora (Dugg.) Hannebert expuestos a soluciones amortiguadoras, ácido acético y $\mathrm{NaOCl}$. Revista Mexicana de Fitopatología. 26: 177-179. 
Samaniego-Gaxiola, J. A. 2008c. Efecto del $\mathrm{pH}$ en la sobrevivencia de esclerocios de Phymatotrichopsis omnivora (Dugg.) Hannebert expuestos a Tilt y Trichoderma sp.

Revista Mexicana de Fitopatología. 26 (1): 32-39.

Samaniego-Gaxiola, J. A. and N. Balagurusamy. 2013. Survival of soil-borne fungus Phymatotrichopsis omnivore after exposure to volatile fatty acids. $J$. Gen Plant Pathol. 79:105-109 https://doi.org/10.1007/s10327-013

Samaniego-Gaxiola, J. A., T. HerreraPérez, A.-Sandoval Pedroza y J. Santamaría-César. 2001. Relaciones entre condiciones de suelo y manejo de huertas de nogal pecanero Carya illinoensis Koch con la dinámica de la pudrición texana, causada por Phymatotrichum omnivorum Duggar. Sociedad Mexicana de Fitopatología .19 (1): 10-18.

Samaniego-Gaxiola, J. A., T. HerreraPérez. 2003. Producción de nuez en nogales [Carya illinoensis (Wangenh.) Koch] atacados por

Phymatotrichopsis omnivora (Duggar) Hennebert. Revista Mexicana de Fitopatología Sociedad Mexicana de Fitoatología. 21(3): 323-330.

Samaniego-Gaxiola, J. A., T. HerreraPérez, A. Pedroza-Sandoval, F. Jiménez-Díaz y Y. I. ChewMadinaveitia. 2003. Fluctuación de la severidad de pudrición texana Phymatotrichopsis omnivora (Duggar) Hennebert en nogal pecanero (Carya illinoenesis K.) bajo las condiciones de la Comarca Lagunera, México.
Revista Mexicana de

Fitopatología. 21(2): 143-151.

Samaniego-Gaxiola, J.A., A. A. FontesPuebla, S. H. Tarango-Rivero y A. Pedroza-Sandoval. (2014)

Comportamiento de la Pudrición

Texana (Phymatotrichopsis omnivora) en Vivero de Nogales.

Revista Mexicana de

Fitopatología. 32 (1): 26-37.

Singleton L.L., D. J. Mihail, and M. C. M. Rush. 2001. Phymatotrichum. In: METHODS FOR RESEARCH ON SOILBORN PHYTOPATHOGENIC FUNGI. APS Press. 3a. Ed. Pp. 145-148. The American Phytopathological Society. St. Paul, Minn.

Sarabia, O. M., P. R. Madrigal, T. M. Martínez, y A. Y. Carreón. 2010. Plantas, hongos micorrízicos y bacterias: su compleja red de interacciones. Biológicas. 12(1):65-71.

Tarango R. S. H. 2006. Fenología y botánica de genotipos de pistachero (pistacia spp.) seleccionados para la formación de portainjertos resistentes al hongo Phymatotrichopsis omnivora (DUGGAR) HENNEBERT. Revista Chapingo Serie Zonas Áridas. 5(1):1-12.

Todd-Watson W, D. Nye-Appel, M. Aloysius-Arnold, Ch. MillerKenerley, J. Lester-Starr and T. Martínez-Trinidad. 2007. Inoculación, síntomas y colonización en árboles de manzano en contenedor por

Phymatotrichopsis omnivora 
(Duggar) Hennebert. Agrociencia. 41(004):459-468.

Uppalapati S. R., S. M. Marek, H.K. Lee, J. Nakashima, Y. Tang, M.K. Sledge, R. A. Dixon and K.

S. Mysore. 2009. Global gene expression profiling during Medicago truncatula Phymatotrichopsis omnivora interaction reveals a role for jasmónica acid, ethylene, and the flavaoonoid pathway in disease development. The American Phytopathological Society. MPMI 22: (1): 7-17.

https://doi.org/10.1094/MPMI-22-

Valero-Galván, J. V., L. N. Muñoz, B. E. Rivera-Chavira, Q. Rascón-Cruz, R. Silva-Vázquez y V. G.

Nevárez-Morillón. 2005. Aislamiento y control de cepas de hongos asociados a la pudrición texana por medio de aceites esenciales de orégano mexicano. XII Congreso Nacional de Biotecnología y Bioingeniería. http://www.smbb.com.mx/congres os\%20smbb/merida05/TRABAJO S/AREA_II/CII-02.pdf.

Vargas-Arispuro, I. C., CorralesMaldonado, S, Vallejo-Cohén y
M. A. Martínez-Téllez. 2005. Correlación entre Contenido de Azufre y Actividad Antifúngica de Variedades de Brassica olerácea

L. para el Control de Phymatotrichopsis omnivora (Duggar) Hennébert. Revista Mexicana de Fitopatología. 23:(3):320-322.

Vargas-Arispuro, I., A. ContrerasValenzuela, J. HernándezMartínez y M. A. Martínez-Téllez. 2006. Arilselenofosfatos con acción antifúngica contra

Phymatotrichopsis omnivora. Revista Fitotecnia Mexicana. Sociedad Mexicana de Fitogenética. 29(2): 1717-174.

Whipps, M. J. 2001. Root exudates as mediators of mineral acquisition in low-nutrient environments. Journal of Experimental Botany. 52:487-511.

https://doi.org/10.1093/jexbot/52.s https://doi.org/10.1093/jxb/52.supp

Zuberer, D.A., C.M. Kenerley and M.J. Jeger. 1988. Populations of bacteria and actinomycetes associated with sclerotia of Phymatotrichum omnivorum buried in Houston black clay. Plant and Soil. 112: 69-76. https://doi.org/10.1007/BF0218175 


\section{Copyright (c) 2016 Lorenza E. Martinez Escudero, Alicia Melgoza Castillo, Ram ona Pérez Leal,}

Anabel Ortega Rodriguez yElizabeth Villalobos Pérez

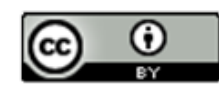

Este tex to está protegido por una licencia licencia Creative Commons 4.0.

Usted es libre para Compartir — copiar y redistribuir el material en cualquier medio o form ato- y Adaptar el documento — remezclar, transformar y crear a partir del material- para cualquier propósito, incluso para fines comerciales, siempre que cumpla la condición de:

Atribución: Usted debe dar crédito a la obra original de manera adecuada, proporcionar un enlace a la licencia, e in dicar si se han realizado cam bios. Puede hacerlo en cualquier form a razonable, pero no de form a tal que sugiera que tiene el apoyo del licenciante o lo recibe por el uso que hace de la obra.

Resumenclelicencia - Textocompletodelalicencia 